\section{Características clínicas de la neumonía adquirida en la comunidad del adulto inmunocompetente hospitalizado según el agente causal}

\author{
FERNANDO SALDÍAS PEÑAFIEL ${ }^{1}$, JAVIERA GASSMANN PONIACHIK ${ }^{\mathrm{a}}$, \\ ALEJANDRO CANELO LÓPEZ ${ }^{a}$, ORLANDO DÍAZ PATIÑO ${ }^{1}$
}

\section{Features of community-acquired pneumonia in immunocompetent hospitalized adults according to the causal agent}

Background: Molecular biological techniques allow the identification of more pathogens associated with community-acquired pneumonia (CAP). Aim: To compare clinical and laboratory parameters of patients with CAP caused by different groups of pathogens. Material and Methods: In a prospective study, immunocompetent adult patients hospitalized with CAP were tested for the presence of a broad range of possible respiratory pathogens using bacterial cultures, polymerase chain reaction, urinary antigen testing and serology. Results: $\mathrm{Pa}$ thogens were detected in 367 of 935 patients with CAP (39.2\%). Streptococcus pneumoniae (10.7\%) and influenza virus (6\%) were the most frequently identified bacterial and viral pathogens, respectively. Pneumococcal pneumonia predominated in older adults, with multiple comorbidities, with elevation of inflammatory parameters and hypoxemia, like other bacterial pneumonias. Viral pneumonia predominated in elderly patients with multiple comorbidities, with a shorter hospital length of stay and lower mortality. Pneumonia associated with atypical microorganisms predominated in young adults, smokers, with subacute clinical evolution. Their hospital stays and lethality was similar to other bacterial pneumonias. Viral and classical bacterial pneumonias predominated in high risk pneumonia severity index categories. Although several variables were associated with the detection of a pathogen group, substantial overlap avoided the identification of reliable clinical predictors to distinguish etiologies. Conclusions: The clinical and radiographic characteristics were similar in pulmonary infections caused by classical bacteria, respiratory viruses and atypical microorganisms. Therefore, microbial testing for common respiratory pathogens is still necessary to optimize treatment.

(Rev Med Chile 2018; 146: 1371-1383)

Key words: Community-Acquired Infections; Diagnosis; Microbiology; Pneumonia.

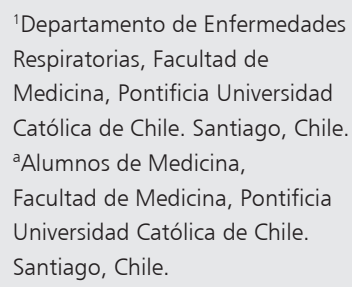

Recibido el 7 de agosto de 2018, aceptado el 26 denoviembre de 2018.

\section{Correspondencia:}

Dr. Fernando Saldías Peñafiel

Departamento de Enfermedades Respiratorias

División de Medicina - Facultad de Medicina

Pontificia Universidad Católica de Chile.

Diagonal Paraguay 362 - Sexto

Piso, Santiago, Chile.

fsaldias@med.puc.cl

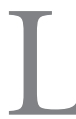

a neumonía adquirida en la comunidad (NAC) es una de las principales enfermedades infecciosas atendidas en los servicios de atención primaria (consultorios y servicios de urgencia), siendo una causa importante de admisión hospitalaria, ocasionando morbilidad y mortalidad significativas ${ }^{1-3}$. Es la principal causa de muerte de origen infeccioso a nivel mundial, con alrededor de tres millones de defunciones por esta causa cada año ${ }^{3}$. La elección del tratamiento antibiótico empírico en las guías clínicas está basado en la gravedad del paciente y el lugar de 
manejo (ambulatorio u hospitalizado); en general, no se considera la información obtenida de la historia clínica para predecir el agente causal de la infección respiratoria ${ }^{1}$. Varios estudios han sugerido que esta información tiene baja sensibilidad y especificidad para predecir la etiología de la infección respiratoria ${ }^{4-14}$.

Streptococcus pneumoniae es el principal agente causal de la NAC en el adulto ${ }^{15-17}$; sin embargo, con las nuevas técnicas microbiológicas basadas en la biología molecular se ha observado un aumento en la frecuencia de infecciones respiratorias ocasionadas por microorganismos atípicos (Mycoplasma pneumoniae, Chlamydophila pneumoniae y Legionella spp) y virus respiratorios ${ }^{18-23}$. En los estudios microbiológicos nacionales, los principales microorganismos aislados son $S$. pneumoniae, $H$. influenzae, microorganismos atípicos y los virus respiratorios; no logrando identificar el agente causal en $40-70 \%$ de los casos debido a las limitaciones de sensibilidad y especificidad de los exámenes microbiológicos tradicionales ${ }^{24-27}$.

El estudio microbiológico de muestras respiratorias basado en las técnicas de biología molecular (reacción en cadena de la polimerasa) y el uso del antígeno urinario de Streptococcus pneumoniae y Legionella pneumophila han facilitado la identificación del agente causal de la neumonía en el adulto hospitalizado, especialmente los virus respiratorios y microorganismos atípicos ${ }^{18-20,28}$. Es importante mencionar que aún no se han desarrollado pruebas diagnósticas rápidas y específicas para $C$. pneumoniae y M. pneumoniae. Sin embargo, la información epidemiológica y las manifestaciones clínicas del paciente con neumonía pueden ser útiles para ampliar la cobertura de la terapia antimicrobiana contra los microorganismos atí$\operatorname{picos}^{4-7,9-13}$. La mayoría de los estudios comparativos son antiguos y no han empleado las nuevas técnicas de biología molecular para identificar el agente infeccioso.

Las recomendaciones de tratamiento antibiótico empírico para el paciente adulto con NAC se basan en los antecedentes epidemiológicos y la gravedad clínica de la enfermedad, en lugar de la presunta etiología, y se recomienda la combinación de antibióticos $\beta$-lactámicos con macrólidos o fluoroquinolonas en los pacientes hospitalizados con NAC ${ }^{29-31}$. Esto implica que la mayoría de los pacientes con neumonía neumocócica o por microorganismos atípicos reciben antibióticos de amplio espectro, lo cual favorece las infecciones con patógenos resistentes a los antibióticos o el desarrollo de resistencia a los antimicrobianos, aumentando los costos de la atención de salud. Idealmente, los pacientes con neumonía neumocócica o por microorganismos atípicos podrían ser tratados con antibióticos de espectro reducido, reduciendo los costos y efectos adversos asociados al tratamiento. El propósito de nuestro estudio fue comparar las características clínicas y de laboratorio en el adulto inmunocompetente hospitalizado por neumonía adquirida en la comunidad según el agente causal (S. pneumoniae, microorganismos atípicos y virus respiratorios).

\section{Pacientes y Métodos}

Estudio clínico descriptivo prospectivo, se evaluaron 935 pacientes adultos hospitalizados por un episodio de neumonía adquirida en la comunidad en el Hospital Clínico de la Pontificia Universidad Católica de Chile entre el 1 de enero de 2014 y 31 de diciembre de 2017. Se enrolaron en el estudio los pacientes adultos inmunocompetentes mayores de 18 años que cumplieron los criterios diagnósticos de neumonía comunitaria descritos por Fang y $\operatorname{cols}^{32}$, quienes aceptaron participar en el estudio previa firma del consentimiento informado. Se excluyeron del estudio a los pacientes menores de 18 años, los portadores del virus de inmunodeficiencia humana, tumores sólidos o hematológicos diagnosticados en los últimos cinco años o en quimioterapia, con neutropenia (menos de 1.000 células $/ \mathrm{mm}^{3}$ ) y tratamiento quimioterápico o inmunosupresor en los seis meses previos a la admisión al hospital. El estudio clínico fue aprobado por el Comité de Ética en Investigación de la Facultad de Medicina de la Pontifiacia Universidad Católica de Chile.

\section{Exámenes microbiológicos}

En los pacientes con neumonía comunitaria hospitalizados se solicitaron los siguientes exámenes microbiológicos: a) Tinción de gram y cultivo de expectoración; b) Hemocultivos aerobios; c) Tinción de gram y cultivo de líquido pleural. La identificación de los agentes microbianos y los estudios de susceptibilidad se realizaron según métodos convencionales ${ }^{33}$. La solicitud de los siguientes exámenes microbiológicos quedó 
a discreción del médico tratante, basados en las características epidemiológicas, evolución clínica y gravedad del caso particular; d) Determinación de antígeno urinario de Streptococcus pneumoniae y Legionella pneumophila serotipo 1 mediante inmunocromatografía (Binax Inc; Scarborough, $\mathrm{ME}$ ); e) Determinación de inmunoglobulinas G y M séricas de Mycoplasma pneumoniae y Chlamydophila pneumoniae en la admisión al hospital y seguimiento a 30 días mediante técnica de inmunofluorescencia indirecta (Zeus Cientific Inc, Raritan NJ). El diagnóstico de infección por microorganismos atípicos estuvo basado en la seroconversión, definida por un aumento de cuatro veces en los títulos de anticuerpos entre los sueros pareados, o en los pacientes que sólo se disponía de suero en la fase aguda por la presencia de un título de $\operatorname{IgM} \geq 1 / 32$ para $M$. pneumoniae $\mathrm{y} \geq 1 / 16$ para C. pneumoniae.

En dos tercios de los pacientes enrolados en el protocolo se tomaron muestras de hisopado nasofaríngeo para la búsqueda de los virus influenza A y B, parainfluenza 1, 2, 3 y 4, virus sincicial respiratorio A y B, adenovirus, metapneumovirus, rhinovirus, coronavirus NL63/229E/OC43, enterovirus y bocavirus. Se empleó la técnica de reacción en cadena de la polimerasa con transcriptasa inversa (RT-PCR) para la detección de antígenos virales específicos (Seeplex RV15 ACE Detection, Seegene, USA).

\section{Información clínica}

En los pacientes enrolados en el estudio se consignaron los siguientes antecedentes clínicos: edad, sexo, comorbilidades, consumo de tabaco y alcohol, uso de antibióticos previo a la hospitalización, lugar de procedencia (hogar, centro geriátrico), sospecha de aspiración bronquial, cuadro clínico de presentación y la presencia de alguna enfermedad aguda concomitante (insuficiencia cardiaca, arritmia, isquemia miocárdica, diabetes descompensada, asma bronquial o enfermedad pulmonar obstructiva crónica reagudizada). Además, se consignó el lugar de manejo (sala de cuidados generales, unidad de cuidado intermedio o cuidados intensivos), los signos vitales y exámenes de laboratorio solicitados en la admisión al hospital (hemograma, proteína $\mathrm{C}$ reactiva, gases arteriales, glicemia, radiografía de tórax, albúmina sérica, electrolitos plasmáticos, nitrógeno ureico y creatinina plasmática) que fueron empleados en el cálculo de los índices de gravedad descritos por Fine y cols. (PSI score) $)^{34}$ y la Sociedad Británica de Tórax (CURB-65) ${ }^{35}$.

Durante la estadía en el hospital se consignaron los tratamientos antimicrobianos y la aparición de las siguientes complicaciones: admisión a UCI, uso de ventilación mecánica, insuficiencia cardiaca congestiva, isquemia miocárdica, arritmias, accidente vascular encefálico, insuficiencia renal aguda, shock séptico, síndrome de dificultad respiratoria aguda, empiema pleural e infección extrapulmonar. La duración de la estadía en el hospital y la sobrevida en el hospital y en el seguimiento a 30 días fueron obtenidos de los registros clínicos.

\section{Análisis estadístico}

Los resultados fueron expresados como valores promedio \pm desviación estándar para las variables medidas en escala numérica y en porcentaje para las medidas en escala nominal. Las variables cualitativas (antecedentes clínico-radiográficos, etiología, evolución clínica y letalidad) fueron comparadas mediante la prueba de $\chi^{2}$ y el test exacto de Fisher, y las variables continuas con la prueba t de Student o análisis de varianza. Para ello se utilizó el programa SPSS versión 23.0. Las diferencias entre las variables fueron consideradas significativas con un valor de $\mathrm{p}<0,05$.

\section{Resultados}

Se enrolaron en el estudio 935 pacientes adultos hospitalizados por neumonía adquirida en la comunidad, edad: $69 \pm 19$ años (rango: 18-102), $51 \%$ sexo masculino, $79 \%$ tenía comorbilidades, un tercio eran fumadores, dos tercios correspondían a las categorías de alto riesgo según Fine y cols. y la Sociedad Británica de Tórax, 90\% fueron tratados con cefalosporinas de tercera generación asociado a macrólidos (19\%), fluoroquinolonas (21\%) o fármacos antianaerobios (14\%), 43\% fueron manejados en unidad de cuidado intermedio o UCI, 11\% requirieron ventilación mecánica, la estadía media en el hospital fueron 9 días (rango: $1-108), 6,3 \%$ fallecieron en el hospital y $8,7 \%$ en el seguimiento a 30 días (Tabla 1).

Los principales microorganismos aislados en los pacientes hospitalizados por neumonía comunitaria fueron Streptococcus pneumoniae (10,7\%), virus influenza $(6 \%)$, virus parainfluenza $(4,4 \%)$, 
Tabla 1. Características clínicas de los pacientes adultos inmunocompetentes hospitalizados por neumonía adquirida en la comunidad

\begin{tabular}{|c|c|}
\hline Variables & $\begin{array}{c}\text { Promedio } \pm \text { DE (Rango) } \\
\text { n (\%) }\end{array}$ \\
\hline $\mathrm{n}$ & 935 adultos \\
\hline Edad (años) & $68,8 \pm 18,7(18-102)$ \\
\hline Sexo (M-F) & $479-456(51-49 \%)$ \\
\hline Fumadores & $345(37 \%)$ \\
\hline Índice de Fine: I-II-III-IV-V & $83-117-156-322-257$ (9-13-17-34-27\%) \\
\hline CURB-65 $\geq 2$ & $608(65 \%)$ \\
\hline Lugar de ingreso: Sala-Intermedio-UCI & 532-210-193 (57-22-21\%) \\
\hline Comorbilidad & $735(79 \%)$ \\
\hline Enfermedad cardiovascular & $317(34 \%)$ \\
\hline Diabetes mellitus & $186(20 \%)$ \\
\hline Enfermedad neurológica crónica & $155(17 \%)$ \\
\hline Enfermedad pulmonar obstructiva crónica & $141(15 \%)$ \\
\hline Insuficiencia renal crónica & 84 (9\%) \\
\hline Neoplasia & $83(9 \%)$ \\
\hline Asma bronquial & $67(7 \%)$ \\
\hline Enfermedad hepática crónica & $27(3 \%)$ \\
\hline Neumonía multilobar & $263(28 \%)$ \\
\hline Derrame pleural & $173(19 \%)$ \\
\hline NAC bacteriémica & $76 \quad(8,1 \%)$ \\
\hline Uso de ventilación mecánica & $105(11 \%)$ \\
\hline Estadía en el hospital (días) & $8,9 \pm 8,3(1-108)$ \\
\hline Complicaciones en el hospital & $381(41 \%)$ \\
\hline Mortalidad en el hospital & $59(6,3 \%)$ \\
\hline Mortalidad a 30 días & $81 \quad(8,7 \%)$ \\
\hline
\end{tabular}

Nota: M: masculino, F: femenino, UCl: Unidad de Cuidados Intensivos, NAC: neumonía adquirida en la comunidad. Los resultados se expresan como valores promedio \pm DE o número de casos y porcentaje.

Haemophilus influenzae (2,8\%), Staphylococcus aureus (2\%), rinovirus (1,6\%), Mycoplasma pneumoniae $(1,5 \%)$ y bacilos gram negativos $(4,8 \%)$. No se logró identificar el agente causal de la neumonía en $60 \%$ de los casos (Tabla 2).

La neumonía neumocócica predominó en adultos mayores, portadores de enfermedades preexistentes, especialmente cardiovasculares, respiratorias y metabólicas crónicas, la duración media de los síntomas antes de la consulta fue seis días, la presencia de hipoxemia, hipoalbuminemia, leucocitosis y proteína $\mathrm{C}$ reactiva elevadas predominaron en este grupo, la estadía media en el hospital fue diez días y 11\% fallecieron en el seguimiento a 30 días. La neumonía asociada a virus respiratorios predominó en adultos mayores con comorbilidad múltiple, la duración media de los síntomas antes de la consulta fue 6,7 días, la estadía media en el hospital fue siete días y $6,1 \%$ fallecieron en el seguimiento a 30 días. La neumonía asociada a microorganismos atípicos predominó en adultos menores de 65 años, fumadores, con comorbilidad cardiovascular, la duración media de los síntomas antes de la consulta fue diez días, 
Tabla 2. Microorganismos aislados en adultos inmunocompetentes hospitalizados por neumonía adquirida en la comunidad

\begin{tabular}{|c|c|c|}
\hline Microorganismos & $\mathbf{n}$ & $\%$ \\
\hline Virus respiratorios & 132 pacientes & 14,1 \\
\hline Influenza A y B & $48-8$ & $5,1-0,9$ \\
\hline Parainfluenza & 41 & 4,4 \\
\hline Rhinovirus spp & 15 & 1,6 \\
\hline Virus sincicial respiratorio & 9 & 1,0 \\
\hline Metapneumovirus & 7 & 0,7 \\
\hline Enterovirus & 4 & 0,4 \\
\hline Coronavirus OC43 & 2 & 0,2 \\
\hline Coronavirus 229E/NL63 & 2 & 0,2 \\
\hline Adenovirus & 2 & 0,2 \\
\hline Bacterias & 215 pacientes & 23,0 \\
\hline Streptococcus pneumoniae & 100 & 10,7 \\
\hline Haemophilus influenzae & 26 & 2,8 \\
\hline Staphylococcus aureus & 19 & 2,0 \\
\hline Mycoplasma pneumoniae & 14 & 1,5 \\
\hline Pseudomonas aeruginosa & 13 & 1,4 \\
\hline Legionella pneumophila & 7 & 0,7 \\
\hline Klebsiella pneumoniae & 6 & 0,6 \\
\hline Chlamydophila pneumoniae & 6 & 0,6 \\
\hline Escherichia coli & 5 & 0,5 \\
\hline Moraxella catarrhalis & 5 & 0,5 \\
\hline Streptococcus spp & 5 & 0,5 \\
\hline Enterobacter cloacae & 2 & 0,2 \\
\hline Serratia marcescens & 2 & 0,2 \\
\hline Enterococcus faecalis & 2 & 0,2 \\
\hline Enterococcus faecium & 1 & 0,1 \\
\hline Klebsiella oxytoca & 1 & 0,1 \\
\hline Proteus mirabilis & 1 & 0,1 \\
\hline Burkholderia cepacia & 1 & 0,1 \\
\hline Stenotrophomonas maltophilia & 1 & 0,1 \\
\hline Morganella morganii & 1 & 0,1 \\
\hline Etiología mixta & 20 pacientes & 2,1 \\
\hline Desconocida & 568 pacientes & 60,8 \\
\hline
\end{tabular}

la estadía media en el hospital fue doce días y $11,5 \%$ fallecieron en el seguimiento a 30 días. Los hallazgos clínicos en los pacientes con neumonía bacteriana no neumocócica fueron similares a lo descrito para la infección respiratoria neumocócica, predominando en adultos mayores con comorbilidad múltiple, especialmente cardiovascular, neurológica, metabólica y respiratoria crónica, la estadía media en el hospital fue más prolongada y menor la sobrevida. En las Tablas
3, 4 y 5 se describen las características sociodemográficas, el cuadro clínico y los exámenes de laboratorio medidos en la admisión al hospital según el agente causal.

Los hallazgos clínicos y radiográficos fueron similares en los diferentes grupos diagnósticos, siendo más frecuente el dolor torácico en la neumonía neumocócica y los escalofríos en las neumonías bacterianas (Tablas 3 y 4, Figura 1). Las neumonías bacterianas y virales predominaron en las categorías de riesgo elevado (índice de gravedad de la neumonía clases IV-V y CURB65 $\geq 2$ ) y las neumonías por microorganismos atípicos en adultos jóvenes con menores factores de riesgo en la admisión al hospital (índice de gravedad de la neumonía clases I-II-III y CURB65 < 2) (Figura 2).

\section{Discusión}

Los principales hallazgos de este estudio fueron: a) Los principales microorganismos aislados en el paciente adulto inmunocompetente hospitalizado por neumonía comunitaria fueron S. pneumoniae, $H$. influenzae, virus respiratorios, microorganismos atípicos y bacilos gram negativos; b) El cuadro clínico radiográfico es similar en la infección pulmonar ocasionada por bacterias clásicas, virus respiratorios y microorganismos atípicos en el adulto hospitalizado por NAC; c) La neumonía neumocócica predominó en adultos mayores, con comorbilidad múltiple, con elevación de parámetros inflamatorios; d) La neumonía viral predominó en adultos mayores con comorbilidad múltiple, siendo menor la estadía en el hospital y la mortalidad; e) La neumonía asociada a microorganismos atípicos predominó en adultos jóvenes, fumadores, con evolución clínica subaguda, siendo la estadía en el hospital y letalidad similar a las otras neumonías bacterianas.

Los exámenes microbiológicos tradicionales asociados a las nuevas técnicas de biología molecular aplicadas a muestras respiratorias han facilitado la identificación del agente causal de la neumonía comunitaria del adulto, especialmente la identificación de los microorganismos atípicos y virus respiratorios ${ }^{19-22}$. Los principales microorganismos aislados en el paciente adulto hospitalizado por neumonía comunitaria fueron S. pneumoniae, $H$. influenzae, virus respiratorios y microorganismos atípicos, similar a lo descrito en 
Tabla 3. Características sociodemográficas y factores de riesgo en el adulto inmunocompetente hospitalizado por neumonía adquirida en la comunidad según el agente causal

\begin{tabular}{|c|c|c|c|c|c|}
\hline Variables & $\begin{array}{c}\text { NAC } \\
\text { neumocócica }\end{array}$ & $\begin{array}{c}\text { Otras } \\
\text { bacterias }\end{array}$ & $\begin{array}{l}\text { Virus } \\
\text { respiratorios }\end{array}$ & $\begin{array}{l}\text { M. pneumoniae } \\
\text { C. pneumoniae }\end{array}$ & $\mathbf{p}$ \\
\hline$n$ & 100 & 89 & 132 & 26 & \\
\hline Edad (años) & $67,1 \pm 17,2$ & $73,6 \pm 16,5$ & $74,1 \pm 16,8^{*}$ & $58,2 \pm 19,6$ & 0,0001 \\
\hline Sexo $(M-F)$ & $\begin{array}{c}58-42 \\
(58-42 \%)\end{array}$ & $\begin{array}{c}56-33 \\
(63-37 \%)\end{array}$ & $\begin{array}{c}58-74 \\
(44-56 \%)\end{array}$ & $\begin{array}{c}16-10 \\
(62-38 \%)\end{array}$ & 0,0236 \\
\hline Fumadores & $40(40 \%)$ & $27(30 \%)$ & $32(24 \%)$ & $13(50 \%)^{* *}$ & 0,0147 \\
\hline Índice de Fine $\geq$ III & $81(81 \%)$ & $83(93 \%)$ & $116(88 \%)$ & $19(73 \%)$ & 0,0181 \\
\hline CURB-65 $\geq 2$ & $74(74 \%)$ & $66(74 \%)$ & $102(77 \%)$ & $12(46 \%)^{* * *}$ & 0,0124 \\
\hline Admisión a UTIM-UCI & $52(52 \%)$ & $50(56 \%)$ & $68(52 \%)$ & $14(54 \%)$ & 0,9132 \\
\hline Comorbilidad & 77 (77\%) & $84(94 \%)$ & $116(88 \%)$ & $16(62 \%)$ & 0,0001 \\
\hline Enfermedad cardiovascular & $35(35 \%)$ & $34(38 \%)$ & $54(41 \%)$ & $8(31 \%)$ & 0,6987 \\
\hline Diabetes mellitus & $18(18 \%)$ & $21(24 \%)$ & $37(28 \%)$ & $4(15 \%)$ & 0,2448 \\
\hline Enfermedad neurológica crónica & 9 (9\%) & $22(25 \%)$ & $27(21 \%)$ & $2(8 \%)$ & 0,0126 \\
\hline Enfermedad pulmonar obstructiva crónica & $21(21 \%)$ & $18(20 \%)$ & $26(20 \%)$ & $2(8 \%)$ & 0,4760 \\
\hline Insuficiencia renal crónica & 9 (9\%) & $7 \quad(8 \%)$ & $20(15 \%)$ & $4(15 \%)$ & 0,2752 \\
\hline Neoplasia & $7 \quad(7 \%)$ & $11(12 \%)$ & $8(6 \%)$ & $2(8 \%)$ & 0,3786 \\
\hline Asma bronquial & $8(8 \%)$ & $5(6 \%)$ & $15(11 \%)$ & $0 \quad(0 \%)$ & 0,1766 \\
\hline Enfermedad hepática crónica & $6(6 \%)$ & 7 (8\%) & $1 \quad(0,8 \%)$ & $0 \quad(0 \%)$ & 0,0277 \\
\hline Hospitalización $\leq 12$ meses & $17(17 \%)$ & $20(22 \%)$ & $28(21 \%)$ & $4(15 \%)$ & 0,7119 \\
\hline Sospecha de aspiración & $6(6 \%)$ & $17(19 \%)^{* * *}$ & $8(6 \%)$ & $3(11 \%)$ & 0,0058 \\
\hline Neumonía multilobar & $34(34 \%)$ & $31(35 \%)$ & $36(27 \%)$ & $6(23 \%)$ & 0,4479 \\
\hline Derrame pleural & $19(19 \%)$ & $18(20 \%)$ & $20(15 \%)$ & $5(19 \%)$ & 0,7720 \\
\hline Uso de ventilación mecánica & $18(18 \%)$ & $21(24 \%)$ & $10(8 \%)^{* * * *}$ & $3(11 \%)$ & 0,0080 \\
\hline Estadía en el hospital (días) & $9,9 \pm 8,2$ & $12,6 \pm 11,1$ & $7,3 \pm 5,5^{* * * * *}$ & $11,9 \pm 11,1$ & 0,0001 \\
\hline Complicaciones en el hospital & $46(46 \%)$ & $41(46 \%)$ & $80(61 \%)$ & $9(35 \%)$ & 0,0231 \\
\hline Mortalidad en el hospital & $10(10 \%)$ & $13(14,6 \%)$ & $5(3,8 \%)^{* * * *}$ & $2(7,7 \%)$ & 0,0419 \\
\hline Mortalidad a 30 días & $11(11 \%)$ & $14(15,7 \%)$ & $8(6,1 \%)$ & $3(11,5 \%)$ & 0,1406 \\
\hline
\end{tabular}

Nota: M: masculino, F: femenino, UTIM: Unidad de Cuidado Intermedio, UCl: Unidad de Cuidados Intensivos, NAC: neumonía adquirida en la comunidad. Los resultados se expresan como valores promedio \pm DE o número de casos y porcentaje. *Comparado con neumonía neumocócica y asociada a microorganismos atípicos. ${ }^{*}$ Comparado con neumonía asociada a virus. ***Comparado con neumonía neumocócica y asociada a virus. ${ }^{* * * *}$ Comparado con neumonía neumocócica y asociada a otras bacterias. ${ }^{* * * *}$ Comparado con neumonía asociada a otras bacterias.

los estudios microbiológicos realizados en nuestro medio y en el extranjero ${ }^{16,17,23-27}$. Las limitaciones de sensibilidad, especificidad, accesibilidad y costos elevados de los exámenes microbiológicos han determinado que en la práctica clínica habitual desconozcamos el agente causal de la neumonía en la mayoría de los casos, por lo cual el tratamien- to antibiótico empírico suele estar basado en la gravedad y lugar de manejo de los enfermos ${ }^{1,16,17}$. Por este motivo, en la década de los 90 se intentó diferenciar clínicamente a los pacientes con neumonía según el agente causal para orientar el tratamiento antibiótico empírico, definiendo dos síndromes: la neumonía típica ocasionada 
Tabla 4. Características clínicas y factores de riesgo en el adulto inmunocompetente hospitalizado por neumonía adquirida en la comunidad según el agente causal

\begin{tabular}{|c|c|c|c|c|c|}
\hline Variables & $\begin{array}{c}\text { NAC } \\
\text { neumocócica }\end{array}$ & $\begin{array}{c}\text { Otras } \\
\text { bacterias }\end{array}$ & $\begin{array}{c}\text { Virus } \\
\text { respiratorios }\end{array}$ & $\begin{array}{l}\text { M. pneumoniae } \\
\text { C. pneumoniae }\end{array}$ & $\mathbf{p}$ \\
\hline $\mathrm{n}$ & 100 & 89 & 132 & 26 & \\
\hline Edad $>65$ años & 59 (59\%) & $67(75 \%)$ & $104(79 \%)$ & $11(42 \%)^{*}$ & 0,0001 \\
\hline Uso previo de antibióticos & $17(17 \%)$ & $30(34 \%)$ & $37(28 \%)$ & $5(19 \%)$ & 0,0480 \\
\hline Duración de los síntomas (días) & $5,8 \pm 4,7$ & $7,2 \pm 6,0$ & $6,7 \pm 8,7$ & $9,9 \pm 7,5^{* *}$ & 0,0645 \\
\hline Fiebre & $70(70 \%)$ & $58(65 \%)$ & $86(65 \%)$ & $20(77 \%)$ & 0,5962 \\
\hline Escalofríos & $30(30 \%)^{* * *}$ & $22(25 \%)$ & $20(15 \%)$ & $5(19 \%)$ & 0,0507 \\
\hline Tos & $81(81 \%)$ & $74(83 \%)$ & $117(89 \%)$ & $21(81 \%)$ & 0,3841 \\
\hline Expectoración & $72(72 \%)$ & $66(74 \%)$ & $88(67 \%)$ & $18(69 \%)$ & 0,6544 \\
\hline Disnea & $73(73 \%)$ & $69(78 \%)$ & $99(75 \%)$ & $22(85 \%)$ & 0,6326 \\
\hline Dolor torácico & $31(31 \%)^{*}$ & $10(11 \%)$ & $26(20 \%)$ & $6(23 \%)$ & 0,0101 \\
\hline Compromiso de conciencia & $25(25 \%)$ & $31(35 \%)$ & $31(23 \%)$ & $4(15 \%)$ & 0,1341 \\
\hline Frecuencia cardiaca (lat/min) & $106,1 \pm 23,3$ & $102,8 \pm 23,4$ & $101,1 \pm 21,4$ & $103,1 \pm 21,6$ & 0,4159 \\
\hline Frecuencia cardiaca > $120 \mathrm{lat} / \mathrm{min}$ & $19(19 \%)$ & $16(18 \%)$ & $23(17 \%)$ & $7(27 \%)$ & 0,7215 \\
\hline Presión arterial sistólica (mmHg) & $117,7 \pm 22,5^{* * *}$ & $121,8 \pm 29,9$ & $128,4 \pm 31,3$ & $117,1 \pm 21,7$ & 0,0217 \\
\hline PA Sistólica < 90 mmHg & $11(11 \%)$ & $11(12 \%)$ & $11(8 \%)$ & $2(8 \%)$ & 0,7504 \\
\hline Presión arterial diastólica (mmHg) & $64,9 \pm 14,0$ & $65,3 \pm 16,0$ & $69,6 \pm 17,5$ & $68,1 \pm 12,8$ & 0,0918 \\
\hline PA Diastólica < 60 mmHg & $34(34 \%)$ & $30(34 \%)$ & $39(30 \%)$ & $7(27 \%)$ & 0,8092 \\
\hline Frecuencia respiratoria (resp/min) & $30,7 \pm 7,6$ & $31,2 \pm 8,8$ & $29,3 \pm 7,0$ & $27,9 \pm 6,5$ & 0,1270 \\
\hline Frecuencia respiratoria $\geq 30 \mathrm{resp} / \mathrm{min}$ & $49(49 \%)$ & $42(47 \%)$ & $55(42 \%)$ & $7(27 \%)$ & 0,1912 \\
\hline Temperatura $\left({ }^{\circ} \mathrm{C}\right)$ & $37,6 \pm 1,1$ & $37,6 \pm 1,0$ & $37,5 \pm 1,0$ & $37,9 \pm 1,0$ & 0,2786 \\
\hline Temperatura $\geq 39{ }^{\circ} \mathrm{C}$ & $12(12 \%)$ & $8(9 \%)$ & $13(10 \%)$ & $7(27 \%)$ & 0,0722 \\
\hline
\end{tabular}

*Comparado con neumonía asociada a virus y otras bacterias. ${ }^{* *}$ Comparado con neumonía neumocócica. ***Comparado con neumonía asociada a virus.

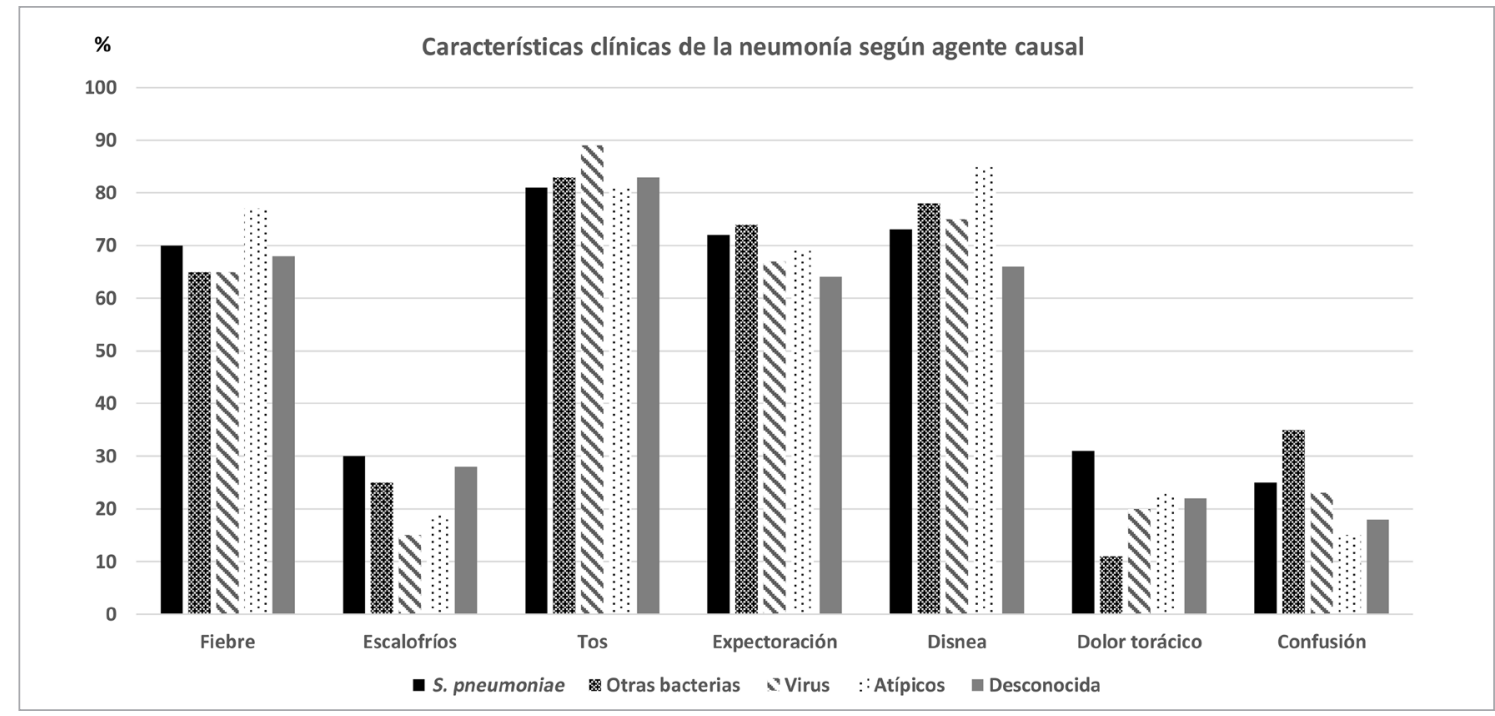

Figura 1. Características clínicas del paciente hospitalizado por neumonía adquirida en la comunidad según agente causal. 
Tabla 5. Exámenes de laboratorio y factores de riesgo en el adulto inmunocompetente hospitalizado por neumonía adquirida en la comunidad según el agente causal

\begin{tabular}{|c|c|c|c|c|c|}
\hline Variables & $\begin{array}{c}\text { NAC } \\
\text { neumocócica }\end{array}$ & $\begin{array}{c}\text { Otras } \\
\text { bacterias }\end{array}$ & $\begin{array}{c}\text { Virus } \\
\text { respiratorios }\end{array}$ & $\begin{array}{l}\text { M. pneumoniae } \\
\text { C. pneumoniae }\end{array}$ & $\mathbf{p}$ \\
\hline$n$ & 100 & 89 & 132 & 26 & \\
\hline Hematocrito (\%) & $39,2 \pm 6,0$ & $37,0 \pm 7,4$ & $38,2 \pm 5,3$ & $37,4 \pm 5,8$ & 0,0951 \\
\hline Hematocrito $<30 \%$ & $6 / 97(6 \%)$ & $11 / 88(13 \%)$ & $11 / 132(8 \%)$ & $3 / 26(12 \%)$ & 0,4716 \\
\hline Leucocitos (por mm³) & $16181 \pm 8314^{*}$ & $12180 \pm 6654$ & $11398 \pm 5864$ & $12795 \pm 6107$ & 0,0001 \\
\hline Leucocitos $\geq 30.000 / \mathrm{mm}^{3}$ & $6 / 97(6 \%)$ & $1 / 88(1 \%)$ & $1 / 132(0,8 \%)$ & $1 / 26(4 \%)$ & 0,0568 \\
\hline Leucocitos $<4.000 / \mathrm{mm}^{3}$ & $3 / 97(3 \%)$ & $5 / 88(6 \%)$ & $3 / 132(2 \%)$ & $1 / 26(4 \%)$ & 0,5963 \\
\hline Neutrófilos (por mm³) & $14362 \pm 7863^{*}$ & $10424 \pm 6281$ & $9437 \pm 5341$ & $11208 \pm 6168$ & 0,0001 \\
\hline Neutrófilos < 1.500/mm³ & $2 / 90(2 \%)$ & $2 / 87(2 \%)$ & $0 / 132(0 \%)$ & $1 / 26(4 \%)$ & 0,2969 \\
\hline Eritrosedimentación (mm/h) & $64,1 \pm 35,9$ & $59,5 \pm 32,8$ & $51,8 \pm 31,9 * *$ & $76,0 \pm 38,7$ & 0,0115 \\
\hline Proteína $\mathrm{C}$ reactiva (mg/dL) & $28,7 \pm 18,3^{* * *}$ & $15,7 \pm 11,2$ & $12,0 \pm 10,2$ & $19,7 \pm 14,9$ & 0,0001 \\
\hline Proteína $C$ reactiva $>50$ mg/dL & $8 / 76(11 \%)^{*}$ & $0 / 80(0 \%)$ & $1 / 130(0,8 \%)$ & $1 / 26(4 \%)$ & 0,0004 \\
\hline Creatininemia (mg/dL) & $1,80 \pm 2,57$ & $1,47 \pm 1,30$ & $1,32 \pm 1,30$ & $1,79 \pm 2,39$ & 0,2420 \\
\hline Creatininemia $>1,5 \mathrm{mg} / \mathrm{dL}$ & 29/96 (30\%) & $24 / 88(27 \%)$ & $30 / 128(23 \%)$ & $6 / 26(23 \%)$ & 0,6872 \\
\hline Nitrógeno ureico sérico (mg/dL) & $31,2 \pm 22,0$ & $29,1 \pm 20,6$ & $26,4 \pm 17,0$ & $30,0 \pm 41,6$ & 0,4429 \\
\hline Nitrógeno ureico sérico $>20 \mathrm{mg} / \mathrm{dL}$ & $57 / 98(58 \%)$ & $46 / 87(53 \%)$ & $77 / 127(61 \%)$ & $8 / 26(31 \%)$ & 0,0399 \\
\hline Glicemia (mg/dL) & $143,0 \pm 62,7$ & $155,6 \pm 76,7$ & $152,0 \pm 72,2$ & $143,3 \pm 77,6$ & 0,6238 \\
\hline Glicemia > 250 mg/dL & $10 / 96(10 \%)$ & $6 / 85(7 \%)$ & $14 / 114(12 \%)$ & $2 / 25(8 \%)$ & 0,6581 \\
\hline Albúmina sérica (g/dL) & $3,32 \pm 0,54$ & $3,24 \pm 0,57$ & $3,63 \pm 0,48 * * * *$ & $3,46 \pm 0,48$ & 0,0001 \\
\hline Albúmina sérica $<3,5 \mathrm{~g} / \mathrm{dL}$ & $54 / 95(57 \%)^{* * * * *}$ & $53 / 84(63 \%)$ & $32 / 112(29 \%)$ & $12 / 25(48 \%)$ & 0,0001 \\
\hline Sodio sérico (mEq/L) & $136,7 \pm 4,6$ & $136,8 \pm 4,9$ & $136,6 \pm 4,5$ & $137,6 \pm 4,8$ & 0,7728 \\
\hline Sodio sérico < $130 \mathrm{mEq} / \mathrm{L}$ & 4/92 (4\%) & $6 / 86(7 \%)$ & $10 / 129(8 \%)$ & $1 / 25(4 \%)$ & 0,7197 \\
\hline Potasio sérico (mEq/L) & $3,83 \pm 0,70 * * * * *$ & $4,08 \pm 0,79$ & $4,18 \pm 0,69$ & $3,89 \pm 0,61$ & 0,0028 \\
\hline Potasio sérico $>5 \mathrm{mEq} / \mathrm{L}$ & $6 / 86(7 \%)$ & $7 / 85(8 \%)$ & $12 / 129(9 \%)$ & $0 / 25(0 \%)$ & 0,4485 \\
\hline $\mathrm{pH}$ arterial & $7,43 \pm 0,07$ & $7,41 \pm 0,09$ & $7,41 \pm 0,06$ & $7,44 \pm 0,09$ & 0,0761 \\
\hline $\mathrm{pH}$ arterial $<7,35$ & $11 / 84(13 \%)$ & $10 / 72(14 \%)$ & $13 / 122(11 \%)$ & $2 / 22(9 \%)$ & 0,8676 \\
\hline $\mathrm{PaO}_{2} / \mathrm{FiO}_{2}$ & $227,7 \pm 89,4^{* * * * * *}$ & $223,7 \pm 86,9$ & $261,2 \pm 74,7$ & $255,9 \pm 78,6$ & 0,0337 \\
\hline $\mathrm{PaO}_{2} / \mathrm{FiO}_{2}<250$ & $44 / 76(58 \%)^{* * * * *}$ & $38 / 63(60 \%)$ & $24 / 65(37 \%)$ & $8 / 19(42 \%)$ & 0,0245 \\
\hline Bicarbonato real (mEq/L) & $21,9 \pm 3,8$ & $21,3 \pm 5,6^{* * * * *}$ & $23,4 \pm 4,1$ & $23,4 \pm 5,8$ & 0,0094 \\
\hline Bicarbonato real < $18 \mathrm{mEq} / \mathrm{L}$ & $13 / 82(16 \%)$ & 20/71 (28\%) & $9 / 121(7 \%)$ & $3 / 22(14 \%)$ & 0,0018 \\
\hline
\end{tabular}

*Comparado con neumonía asociada a virus y otras bacterias. ${ }^{* *}$ Comparado con neumonía asociada a microorganismos atípicos. ${ }^{* *}$ Comparado con neumonía asociada a otras bacterias, virus y microorganismos atípicos. ****Comparado con neumonía neumocócica o asociada a otras bacterias. ${ }^{* * * *}$ Comparado con neumonía asociada a virus. ${ }^{* * * * *}$ Comparado con neumonía asociada a virus y microorganismos atípicos. 


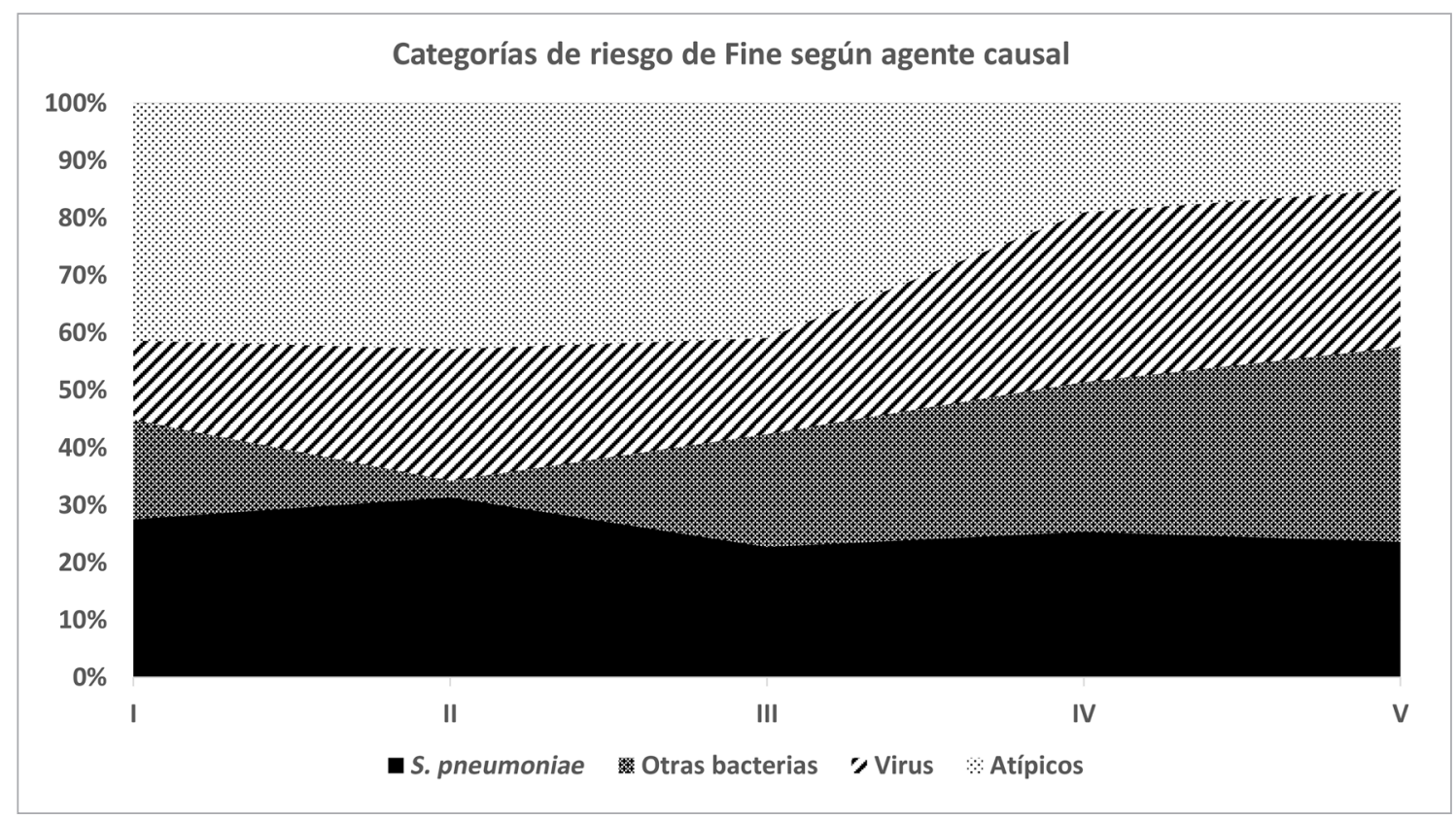

Figura 2. Categorías de riesgo de Fine según el agente causal de la neumonía adquirida en la comunidad.

por S. pneumoniae, H. influenzae y bacilos gram negativos que pueden ser manejados con agentes $\beta$-lactámicos y la neumonía atípica ocasionada por M. pneumoniae, C. pneumoniae y L. pneumophi$l a$, en quienes se recomienda el tratamiento con macrólidos, tetraciclinas of fluoroquinolonas ${ }^{1,36,37}$.

Originalmente se había planteado que las infecciones respiratorias ocasionadas por virus respiratorios y microorganismos atípicos (Mycoplasma pneumoniae, Chlamydophila pneumoniae) podían ser diferenciadas por el cuadro clínico y la radiografía de tórax de la neumonía neumocócica ${ }^{36,37}$. Estos microorganismos ocasionarían síntomas y signos fácilmente reconocibles, lo que llevó a acuñar el concepto de neumonía atípica. En este síndrome, la producción de esputo, leucocitosis y la condensación pulmonar en las radiografías de tórax eran inusuales, las manifestaciones extrapulmonares eran comunes, especialmente dermatitis, complicaciones neurológicas, hepatitis, alteraciones cardiovasculares y disfunción renal ${ }^{36-41}$. Por definición, los exámenes microbiológicos tradicionales de esputo y sangre eran estériles.

En nuestro estudio los hallazgos clínicos, radiográficos y exámenes de laboratorio medidos en la admisión al hospital no permitieron identificar con precisión y certeza al agente causal de la infección respiratoria, lo cual ha sido comunicado en otros estudios ${ }^{4-14}$. Se identificaron algunos elementos clínicos diferenciadores, tales como el predominio de infecciones respiratorias por microorganismos atípicos en adultos jóvenes con cuadro clínico de evolución subaguda o el mejor pronóstico observado en pacientes con neumonía asociada a virus respiratorios ${ }^{36-41}$. En la Tabla 6 se enumeran los principales elementos clínicos que permiten diferencia la infección pulmonar asociada a bacterias clásicas y microorganismos atípicos descritos en la literatura. Sin embargo, la superposición de hallazgos clínicos es significativa, lo cual impide precisar el agente causal de la neumonía basado exclusivamente en elementos clínicos. De hecho, se han examinado varias reglas predictivas en la literatura que han demostrado pobre capacidad predictiva para precisar el agente causal de la infección pulmonar ${ }^{9-14}$. Por este motivo, las guías de práctica clínica de neumonía comunitaria han abandonado la aproximación sindromática y sugieren abandonar el término neumonía atípica para evitar confusión ${ }^{29-31}$.

Las neumonías asociadas a bacterias clásicas $y$ virus respiratorios en el adulto inmunocompe- 


\section{Tabla 6. Elementos clínicos y radiográficos diferenciadores en pacientes adultos con neumonía adquirida en la comunidad según el agente causal}

\begin{tabular}{|c|c|}
\hline Publicación, año & Elementos clínicos y radiográficos diferenciadores según el agente causal \\
\hline $\begin{array}{l}\text { Yu VL et al. }{ }^{4} \\
1982\end{array}$ & L. pneumophila: El cuadro clínico es inespecífico, siendo más frecuente la hiponatremia \\
\hline $\begin{array}{l}\text { Woodhead MA } € \\
1987\end{array}$ & $\begin{array}{l}\text { M. pneumoniae: Adultos jóvenes, síntomas tracto respiratorio alto, ausencia de leucocitosis y } \\
\text { síntomas sistémicos } \\
\text { S. pneumoniae y Legionella spp: Fiebre elevada, compromiso de conciencia, síntomas sistémicos, } \\
\text { leucocitosis }\end{array}$ \\
\hline $\begin{array}{l}\text { Farr BM et al. }{ }^{10} \\
1989\end{array}$ & $\begin{array}{l}\text { NAC neumocócica: Edad avanzada, evolución aguda, esputo sanguinolento, dolor torácico, } \\
\text { leucocitosis y compromiso radiográfico lobar }\end{array}$ \\
\hline $\begin{array}{l}\text { Granados A et al. }{ }^{11} \\
1989\end{array}$ & L. pneumophila: Cuadro clínico inespecífico no permite diferenciarlo de la NAC neumocócica \\
\hline $\begin{array}{l}\text { Kauppinen MT } \epsilon \\
1996\end{array}$ & $\begin{array}{l}\text { C. pneumoniae: Evolución subaguda, presencia de cefalea, menor producción de esputo, pará- } \\
\text { metros inflamatorios bajos }\end{array}$ \\
\hline $\begin{array}{l}\text { Lieberman D et } \\
1996\end{array}$ & L. pneumophila: Cuadro clínico inespecífico no permite diferenciarlo de otros agentes causales \\
\hline $\begin{array}{l}\text { Marrie TJ et al. } \\
1996\end{array}$ & $\begin{array}{l}\text { Microorganismos atípicos: Cuadro clínico inespecífico no permite diferenciarlos de otros agentes } \\
\text { causales }\end{array}$ \\
\hline $\begin{array}{l}\text { Molinos L et al. }{ }^{8} \\
1997\end{array}$ & $\begin{array}{l}\text { NAC bacteriana clásica: Evolución aguda, expectoración purulenta, dolor torácico, condensación } \\
\text { pulmonar, leucocitosis }\end{array}$ \\
\hline $\begin{array}{l}\text { Tan MJ et al. }{ }^{12} \\
2000\end{array}$ & $\begin{array}{l}\text { L. pneumophila: Características radiográficas inespecíficas no permiten diferenciarlo de otros } \\
\text { agentes causales }\end{array}$ \\
\hline $\begin{array}{l}\text { Ruiz-González A et al. }{ }^{42} \\
2000\end{array}$ & $\begin{array}{l}\text { NAC bacteriana vs viral: Evolución aguda, adultos mayores de } 65 \text { años, presencia de comorbili- } \\
\text { dades, leucopenia o leucocitosis }\end{array}$ \\
\hline $\begin{array}{l}\text { Fernández-Sabé N } \\
\text { et al. }{ }^{43} 2003\end{array}$ & $\begin{array}{l}\text { L. pneumophila: Sexo masculino, fiebre elevada, ausencia de dolor torácico y esputo purulento } \\
\text { Cuadro clínico inespecífico no permite diferenciarlo de otros agentes causales }\end{array}$ \\
\hline $\begin{array}{l}\text { Schneeberger PM et al. }{ }^{44} \\
2004\end{array}$ & $\begin{array}{l}\text { Microorganismos atípicos: Predominaron en el adulto joven, pero el cuadro clínico fue inespe- } \\
\text { cífico }\end{array}$ \\
\hline $\begin{array}{l}\text { Sopena } \mathrm{N} \text { et al. }{ }^{13} \\
2004\end{array}$ & $\begin{array}{l}\text { S. pneumoniae: Presencia de comorbilidades, tos y expectoración } \\
\text { C. pneumoniae: Edad avanzada, antecedente de EPOC, evolución subaguda } \\
\text { L. pneumophila: Adultos jóvenes sin comorbilidades, ingesta etílica, presencia de cefalea, diarrea, } \\
\text { hiponatremia }\end{array}$ \\
\hline $\begin{array}{l}\text { Boersma WG et al. }{ }^{45} \\
2006\end{array}$ & $\begin{array}{l}\text { M. pneumoniae: Opacidades alveolares en parche } \\
\text { C. pneumoniae: Compromiso radiográfico unilobar }\end{array}$ \\
\hline $\begin{array}{l}\text { Marcos A et al. }{ }^{46} \\
2006\end{array}$ & NAC viral vs bacteriana: El único elemento diferenciador fue el recuento de leucocitos \\
\hline $\begin{array}{l}\text { Jennings LC et al. }{ }^{38} \\
2008\end{array}$ & $\begin{array}{l}\text { NAC viral vs bacteriana: Cuadro clínico inespecífico, las mialgias fueron más frecuentes en la } \\
\text { NAC viral }\end{array}$ \\
\hline $\begin{array}{l}\text { Johnstone J et al. }{ }^{47} \\
2008\end{array}$ & $\begin{array}{l}\text { NAC viral vs bacteriana: Cuadro clínico inespecífico, adultos mayores frágiles con comorbilidad } \\
\text { cardiovascular fueron más frecuentes en la NAC viral }\end{array}$ \\
\hline $\begin{array}{l}\text { Lui G et al. }{ }^{39} \\
2009\end{array}$ & Microorganismos atípicos: Cuadro clínico inespecífico y gravedad similar a otros agentes causales \\
\hline $\begin{array}{l}\text { Huijskens EG et al. }{ }^{14} \\
2014\end{array}$ & $\begin{array}{l}\text { Virus respiratorios: Edad avanzada y tos } \\
\text { NAC grave: Aumenta el rendimiento de los exámenes microbiológicos }\end{array}$ \\
\hline $\begin{array}{l}\text { Huijts SM et al. }{ }^{48} \\
2014\end{array}$ & $\begin{array}{l}\text { NAC neumocócica: Presencia de tos, dolor torácico, inicio agudo de los síntomas, parámetros } \\
\text { inflamatorios elevados }\end{array}$ \\
\hline
\end{tabular}


tente hospitalizado presentaron mayores factores de riesgo y gravedad en la admisión al hospital comparado con las neumonías asociadas a microorganismos atípicos (Mycoplasma pneumoniae y Chlamydophila pneumoniae) que predominaron en adultos jóvenes previamente sanos con escasas comorbilidades. A pesar de ello, la estadía y mortalidad en el hospital fueron menores en los pacientes con neumonía asociada a virus respiratorios. Es probable que los índices predictores de riesgo, tales como el índice de gravedad de la neumonía, CURB-65 y SCAP, tengan diferente capacidad de predecir los eventos adversos en la evolución en el hospital según el agente causal de la infección pulmonar.

En conclusión, las manifestaciones clínicas, hallazgos de la radiografía de tórax y exámenes de laboratorio solicitados en la admisión al hospital no permitieron identificar con precisión el agente causal de la infección pulmonar en el adulto inmunocompetente. Existen algunos elementos diferenciadores pero carecen de sensibilidad y especificidad para orientar al clínico en la elección del tratamiento antimicrobiano empírico. Los exámenes microbiológicos basados en las técnicas de biología molecular de muestras respiratorias han facilitado la identificación del agente causal de la infección pulmonar; sin embargo, la complejidad técnica y elevado costo de los exámenes ha circunscrito su empleo principalmente en los pacientes hospitalizados con neumonía comunitaria grave.

\section{Referencias}

1. Gotfried M, Freeman C. An update on community-acquired pneumonia in adults. Compr Ther 2000; 26: 283-93.

2. Marston BJ, Plouffe JF, File TM, Hackman BA, Salstrom SJ, Lipman HB, et al. Incidence of community-acquired pneumonia requiring hospitalization. Results of a population- based active surveillance study in Ohio. The Community-Based Pneumonia Incidence Study Group. Arch Intern Med 1997; 157: 1709-18.

3. GBD 2015 LRI Collaborators. Estimates of the global, regional, and national morbidity, mortality, and aetiologies of lower respiratory tract infections in 195 countries: a systematic analysis for the Global Burden of Disease Study 2015. Lancet Infect Dis 2017; 17: 1133-61.

4. Yu VL, Kroboth FJ, Shonnard J, Brown A, McDearman
S, Magnussen M. Legionnaire's disease: new clinical perspective from a prospective pneumonia study. Am J Med 1982; 73: 357-61.

5. Kauppinen MT, Saikku P, Kujala P, Herva E, Syrjälä $\mathrm{H}$. Clinical picture of community-acquired Chlamydia pneumoniae pneumonia requiring hospital treatment: a comparison between chlamydial and pneumococcal pneumonia. Thorax 1996; 51: 185-9.

6. Lieberman D, Porath A, Schlaeffer F, Lieberman D, Boldur I. Legionella species community-acquired pneumonia. A review of 56 hospitalized adult patients. Chest 1996; 109: 1243-9.

7. Marrie TJ, Peeling RW, Fine MJ, Singer DE, Coley CM, Kapoor WN. Ambulatory patients with community-acquired pneumonia: The frequency of atypical agents and clinical course. Am J Med 1996; 101: 508-15.

8. Molinos L, Fernández R, Gullón JA, Rubines G, Alonso MA, Escudero C, et al. Neumonía adquirida en la comunidad (NAC) con tratamiento hospitalario. Interés de la clínica y exámenes complementarios en la predicción de la etiología. Arch Bronconeumol 1997; 33: 230-4.

9. Woodhead MA, MacFarlane JT. Comparative clinical and laboratory features of Legionella with pneumococcal and mycoplasma pneumonias. Br J Dis Chest 1987; 81: 133-9.

10. Farr BM, Kaiser DL, Harrison BD, Connolly CK. Prediction of microbial aetiology at admission to hospital for pneumonia from the presenting clinical features. Thorax 1989; 44: 1031-5.

11. Granados A, Podzamczer D, Gudiol F, Manresa F. Pneumonia due to Legionella pneumophila and pneumococcal pneumonia: similarities and differences on presentation. Eur Respir J 1989; 2: 130-4.

12. Tan MJ, Tan JS, Hamor RH, File TM Jr, Breiman RF. The radiologic manifestations of Legionnaire's disease. The Ohio Community-Based Pneumonia Incidence Study Group. Chest 2000; 116: 398-403.

13. Sopena N, Pedro-Botet ML, Sabrià M, García-Parés D, Reynaga E, García-Núñez M. Comparative study of community-acquired pneumonia caused by Streptococcus pneumoniae, Legionella pneumophila or Chlamydia pneumoniae. Scand J Infect Dis 2004; 36: 330-4.

14. Huijskens EG, Koopmans M, Palmen FM, van Erkel AJ, Mulder PG, Rossen JW. The value of signs and symptoms in differentiating between bacterial, viral and mixed aetiology in patients with community-acquired pneumonia. J Med Microbiol 2014; 63 (Pt 3): 441-52.

15. Isturiz RE, Luna CM, Ramírez J. Clinical and economic burden of pneumonia among adults in Latin America. Int J Infect Dis 2010; 14: e852-6.

16. Torres A, Blasi F, Peetermans WE, Viegi G, Welte T. 
The aetiology and antibiotic management of community-acquired pneumonia in adults in Europe: a literature review. Eur J Clin Microbiol Infect Dis 2014; 33: 106579.

17. Peto L, Nadjm B, Horby P, Ngan TT, van Doorn R, Van Kinh N, et al. The bacterial aetiology of adult community-acquired pneumonia in Asia: a systematic review. Trans R Soc Trop Med Hyg 2014; 108: 326-37.

18. Daxboeck F, Krause R, Wenisch C. Laboratory diagnosis of Mycoplasma pneumoniae infection. Clin Microbiol Infect 2003; 9: 263-73.

19. Chan YR, Morris A. Molecular diagnostic methods in pneumonia. Curr Opin Infect Dis 2007; 20: 157-64.

20. Nolte FS. Molecular diagnostics for detection of bacterial and viral pathogens in community-acquired pneumonia. Clin Infect Dis 2008; 47 (Suppl 3): S123-6.

21. Johansson N, Kalin M, Tiveljung-Lindell A, Giske CG, Hedlund J. Etiology of community-acquired pneumonia: increased microbiological yield with new diagnostic methods. Clin Infect Dis 2010; 50: 202-9.

22. Wu X, Wang Q, Wang M, Su X, Xing Z, Zhang W, et al. Incidence of respiratory viral infections detected by PCR and real-time PCR in adult patients with community-acquired pneumonia: a meta-analysis. Respiration 2015; 89: 343-52.

23. Alimi Y, Lim WS, Lansbury L, Leonardi-Bee J, Nguyen-Van-Tam JS. Systematic review of respiratory viral pathogens identified in adults with community-acquired pneumonia in Europe. J Clin Virol 2017; 95: 26-35.

24. Riquelme R, Riquelme M, Rioseco ML, Gómez V, Gil $\mathrm{R}$, Torres A. Etiología y factores pronósticos de la neumonía adquirida en la comunidad en el adulto hospitalizado, Puerto Montt, Chile. Rev Med Chile 2006; 134: 597-605.

25. Díaz A, Barría P, Niederman M, Restrepo MI, Dreyse J, Fuentes G, et al. Etiology of community-acquired pneumonia in hospitalized patients in Chile: the increasing prevalence of respiratory viruses among classic pathogens. Chest 2007; 131: 779-87.

26. Luchsinger V, Ruiz M, Zunino E, Martínez MA, Machado C, Piedra PA, et al. Community-acquired pneumonia in Chile: the clinical relevance in the detection of viruses and atypical bacteria. Thorax 2013; 68: 1000-6.

27. Saldías F, Ortega M, Fuentes G, Elola JM, Uribe J, Morales A, et al. Participación de los virus respiratorios en la neumonía del adulto inmunocompetente adquirida en la comunidad. Rev Med Chile 2016; 144: 1513-22.

28. Sinclair A, Xie X, Teltscher M, Dendukuri N. Systematic review and meta-analysis of a urine-based pneumococcal antigen test for diagnosis of community-acquired pneumonia caused by Streptococcus pneumoniae. J Clin Microbiol 2013; 51: 2303-10.

29. Mandell LA, Wunderink RG, Anzueto A, Bartlett JG, Campbell GD, Dean NC, et al; Infectious Diseases Society of America; American Thoracic Society. Infectious Diseases Society of America/American Thoracic Society consensus guidelines on the management of community-acquired pneumonia in adults. Clin Infect Dis 2007; 44 (Suppl 2): S27-72.

30. Lim WS, Baudouin SV, George RC, Hill AT, Jamieson C, Le Jeune I, et al. Pneumonia Guidelines Committee of the British Thoracic Society Standards of Care Committee. British Thoracic Society guidelines for the management of community acquired pneumonia in adults: update 2009. Thorax 2009; 64 (Suppl 3): 1-55.

31. Bantar C, Curcio D, Jasovich A, Bagnulo H, Arango A, Bavestrello L, et al. Neumonía aguda adquirida en la comunidad en adultos: Actualización de los lineamientos para el tratamiento antimicrobiano inicial basado en la evidencia local del Grupo de Trabajo de Sudamérica (ConsenSur II). Rev Chilena Infectol 2010;27(Suppl 1):S9-S38.

32. Fang GD, Fine M, Orloff J, Arisumi D, Yu VL, Kapoor $W$, et al. New emerging etiologies for community-acquired pneumonia with implications for therapy. A prospective multicenter study of 359 cases. Medicine (Baltimore) 1990; 69: 307-16.

33. Baron E, Murray P. Bacteriology. In: Murray P, Baron E, Pfaller M, Tenover F, Yolken R, eds. Manual of Clinical Microbiology. Washington DC: ASM Press, 1999; 246821.

34. Fine MJ, Auble TE, Yealy DM, Hanusa BH, Weissfeld LA, Singer DE, et al. A prediction rule to identify lowrisk patients with community-acquired pneumonia. $\mathrm{N}$ Engl J Med 1997; 336: 243-50.

35. Lim WS, Van Der Eerden MM, Laing R, Boersma WG, Karalus N, Town GI, et al. Defining community acquired pneumonia severity on presentation to hospital: an international derivation and validation study. Thorax 2003; 58: 377-82.

36. Martin RE, Bates JH. Atypical pneumonia. Infect Dis Clin North Am 1991; 5: 585-601.

37. Cunha BA. The atypical pneumonias: clinical diagnosis and importance. Clin Microbiol Infect 2006; 12 (Suppl 3): $12-24$.

38. Jennings LC, Anderson TP, Beynon KA, Chua A, Laing RT, Werno AM, et al. Incidence and characteristics of viral community-acquired pneumonia in adults. Thorax 2008; 63: 42-8.

39. Lui G, IP M, Lee N, Rainer TH, Man SY, Cockram CS, et al. Role of 'atypical pathogens' among adult hospitalized 
patients with community-acquired pneumonia. Respirology 2009; 14: 1098-105.

40. Basarab M, Macrae MB, Curtis CM. Atypical pneumonia. Curr Opin Pulm Med 2014; 20: 247-51.

41. Kishaba T. Community-acquired pneumonia caused by Mycoplasma pneumoniae: how physical and radiological examination contribute to successful diagnosis. Front Med (Lausanne) 2016; 3: 28.

42. Ruiz-González A, Falguera M, Vives M, Nogués A, Porcel JM, Rubio-Caballero M. Community-acquired pneumonia: development of a bedside predictive model and scoring system to identify the aetiology. Respir Med 2000; 94: 505-10.

43. Fernández-Sabé N, Rosón B, Carratala J, Dorca J, Manresa F, Gudiol F. Clinical diagnosis of Legionella pneumonia revisited: evaluation of the Community-Based Pneumonia Incidence Study Group scoring system. Clin Infect Dis 2003; 37: 483-9.

44. Schneeberger PM, Dorigo-Zetsma JW, van der Zee A, van Bon M, van Opstal JL. Diagnosis of atypical patho- gens in patients hospitalized with community-acquired respiratory infection. Scand J Infect Dis 2004; 36: 26973.

45. Boersma WG, Daniels JM, Löwenberg A, Boeve WJ, van de Jagt EJ. Reliability of radiographic findings and the relation to etiologic agents in community-acquired pneumonia. Respir Med 2006; 100: 926-32.

46. Angeles M, Camps M, Pumarola T, Martínez JA, Martínez E, Mensa J, et al. The role of viruses in the aetiology of community-acquired pneumonia in adults. Antivir Ther 2006; 11: 351-9.

47. Johnstone J, Majumdar SR, Fox JD, Marrie TJ. Viral infection in adults hospitalized with community-acquired pneumonia: prevalence, pathogens, and presentation. Chest 2008; 134: 1141-8.

48. Huijts SM, Boersma WG, Grobbee DE, Gruber WC, Jansen KU, Kluytmans JW, et al. Predicting pneumococcal community-acquired pneumonia in the emergency department: evaluation of clinical parameters. Clin Microbiol Infect 2014; 20: 1316-22. 